\title{
Index
}

Adenauer, Konrad 14, 16, 51-54, 56-57, 59-60, 62-67, 69-71, 77-80, 84, 92-93, 148-149, 159, 168, 177

Adorno, Theodor 8, 10-11, 26, 31-32, 35, 37, 42, 127, 151, 154-155, 180, 182,198

Aho, Esko 96

Allied Marine Transportation Executive see AMTE

America see US

AMTE (Allied Marine Transportation Executive) 55, 57

Anglo-French Coordinating Committee 57

Arendt, Hannah 8, 17, 34, 80, 141, 152, 158, 173-175

Arendtian 60, 63

Auschwitz 16, 33, 35-36, 40, 78, 90, 94, 97, 107

Austria 95-96

\section{Belgium 56, 65}

Benhabib, Seyla 3, 11, 39, 156, 174, 190

Benjamin, Walter 8, 26-30, 32-37, 40, 69

'Theses on the Philosophy of History' 32-34, 37, 40

Berlusconi, Silvio 115

Blair, Tony 155

Bodin, Jean 64

Brandt, Willy 90

Brexit 2, 16-18, 79, 109-110, 124-130, 142

Bulgaria 110-111
CAP (Common Agricultural Policy) 84-85

Catholic 55, 59-60, 63, 80, 89, 110

GFLN (Comité français de la liberation nationale) 58, 82

Chirac, Jacques 112, 123

Christian, Christianity 60, 69, 94, 128, 190

Churchill, Winston 82, 86, 126

Civil War (American) 18, 169, 172, 178-179, 182

classic narrative of integration 14-16, 77, 79, 90-91, 97-99, 107-108, 110, 116, 120, 129-130, 168, 173

Cold War 25, 53, 68, 78, 90, 95, 97, 99, 108, 110-111, 117, 122, 125, 146, 168,196

Comité français de la liberation nationale see GFLN

Common Agricultural Policy see CAP

Coudenhove-Kalergi, Richard von 35

Council of Europe 88

crisis of the Eurozone see Eurozone crisis

critical theory see Frankfurt School

Croatia 115, 122

Delors, Jacques 16, 71, 79, 91-95, 141, 149, 159, 168, 170

Denmark 79, 87-88

Derrida, Jacques 4, 7, 15, 77, 98, 107

ECB (European Central Bank) 91, 118-119, 142, 144-147, 158

Eco, Umberto 155, 157

Economic and Monetary Union see EMU 


\section{Index}

ECSC (European Coal and Steel

Community) 1, 14-15, 51, 53-54, 61-70, 85-86, 91, 106, 144, 167

EDC (European Defense Community) 70, 91

EMU (Economic and Monetary Union) 16, 91-94, 120, 142, 146-147, 150, 159, 180-181, 197

Entente 55, 58

Erdoğan, Recep Tayyip 123

Erhard, Ludwig 66

Euratom 71, 83 euro 2, 91, 117-120, 142, 145, 149, 158, 167,180

European Central Bank see EGB

European Coal and Steel Community see ECSC

European Defense Community see EDC Eurozone crisis 2, 16, 98-89, 108, 110, 117-121, 124, 129-131, 142, 144-148, 150, 153, 158-159, 168, 179-181, 192, 194-195, 197, 200

Farage, Nigel 99, 125, 128

fascism 2, 82, 99, 115-116, 127, 129, 147, 151, 168, 190-191, 195, 198

Fini, Gianfranco 115

Finland 95-96, 190

Fischer, Joschka 112

Fouchet, Christian 83

Fouchet Plan 83, 85

Franco, Francisco 88-89

Franco-Prussian War 55

Frankfurt School (of critical theory) 3, 5, 8, 9-13, 26-44, 119, 127, 131, 141-143, 150-155, 171, 180, 182-184, 189, 190-191, 198-199

Gabriel, Sigmar 192-193

Gaulle, Charles de 15-16, 71, 78-85, 87-88, 187

Gaullist 15, 109

generations 3-4, 6, 12, 26, 28, 36-37, 39, 43-44, 64, 78-80, 94, 98-99, 109, 114, 125, 127, 129, 141, 143, 146-153, 156, 158, 169-178, 181, 188-192

Genscher, Hans-Dietrich 112
González, Felipe 89

Gorbachev, Mikhail 94

Great Britain see UK

Great Depression 2, 99, 117, 146-147, 150, 172, 189, 191, 195, 198

Great Recession 2, 13, 16, 99, 117-118, 125, 128, 130, 147, 150, 169, 182, 188, 190-192, 194, 198-200

Greece 2, 25, 88-90, 95, 108-109, 117 , 119, 144-147, 161, 190

Guisan, Catherine 5, 7-8, 13-14, 27, 52

HA (High Authority) 4, 58, 61, 65-66

Haas, Ernst 5, 7, 86

Habermas, Jürgen 8, 15, 26, 32, 35, 37-40, 77, 98, 107, 142-143, 146, 149-150, 155, 173-175, 179-181, 192-193, 196-197

Halbwachs, Maurice 29-31, 34

Hallstein, Walter 66, 84-85, 122

Heath, Edward 87

Hegel, G. W. F. 8, 34

High Authority see HA

Hitler, Adolf 89, 95, 112, 145-146, 149-150

Hoffman, Stanley 80, 86, 120

Holocaust 16, 26, 35, 77-78, 90, 94, 97-98, 107-108, 112-116, 122, 128-129, 156

Horkheimer, Max 3, 8, 11, 26, 28-30, $35,142,149,151,182,187$, 198-199

Hume, David 170

Hungary 2, 99, 109, 128, 147

Institute for Social Research (Institut für Sozialforschung) see Frankfurt School

Iraq 15, 98

Ireland 79, 87-88, 117, 144

Italy 2, 55, 65, 77, 82, 115, 128, 145, 147, 161,190

Jaspers, Karl 8

Jefferson, Thomas 156

Judt, Tony 106-108

Kaczyński, Jarosław Aleksander 128

Kalniete, Sandra 114 


\section{Index}

Karamanlis, Konstantinos 89

Kellogg-Briand Pact 35, 80

Keynes, John Maynard 58

Kohl, Helmut 16, 71, 79, 91-95, 144-150, 159, 168

Krenz, Egon 94

Kundera, Milan 113

Le Pen, Marine 127-128

League of Nations 35, 53, 57, 64

Lincoln, Abraham 18, 167, 169, 176-179

Luxembourg Compromise 85, 88

Macmillan, Harold 86-87

Mannheim, Karl 36-37

Marcuse, Herbert 8, 11, 26, 34, 37-38, 151-153, 161, 171,199

Marshall Plan 68, 93, 126

Marx, Karl 38, 152

Merkel, Angela 145, 150, 154

Milward, Alan 14-15

Mitterrand, François 16, 71, 79, 91-95, 149, 159, 168

Monnet, Jean 14, 16, 51, 53-58, 60-71, 77-83, 91-93, 99, 110, 142, 148-149, 151, 159, 168

Mussolini, Benito 77, 115

Nathan, Robert 58

nationalism 1-2, 4, 13-14, 54, 56, 60, 62, 69, 77, 80-81, 83-84, 88, 94, 98, 109, 142-143, 146-147, 149, 178, 183, 187, 189-190

NATO (North Atlantic Treaty

Organization) 83, 89-90, 126

Netherlands 65, 127, 144

Dutch 56, 66, 68, 84

Nietzsche, Friedrich 26, 42

Nixon, Richard 83

Offe, Claus 119, 142, 153

Orbán, Viktor 99, 128

Parsons, Craig 15, 53

Plan de modernisation et d'equipement 61, 63, 83, 92
Poland 2, 57, 99, 109, 112-113, 128-129, 147

Pollock, Friedrich 198-199

Pompidou, Georges 87

Publius 171-172, 175

Portugal 25, 88-90, 117, 122, 144

postcommunist 7, 16, 95-98, 108-117, 121, 123-124, 128-129, 131, 148-149, 153, 168, 194

Rallis, George 89

Reynaud, Paul 59, 82

Roman Catholic see Catholic

Romania 110-111

Rousso, Henry 25

Salazar, António de Oliveira 88

Sarkozy, Nicolas 120, 123

Sartre, Jean-Paul 149

Schröder, Gerhard 150

Schumacher, Kurt 67

Schuman Declaration, Schuman Plan 15, 17, 62-69, 84, 148, 195

Schuman, Robert 14, 16, 51, 53-57, 59-67, 69-71, 77-80, 92-93, 148-149, 159, 168, 177

Serbia 124

Sierp, Aline 8-9, 27

Single European Act 149

Soares, Mário 90

Slovenia 115-116, 122

Spain 25, 88-90, 117, 122, 144, 190

Stalin, Joseph 67, 120, 150

Sweden 95-96

Switzerland 86

Szájer, Josef 115

TEU see Treaty on European Union Thatcher, Margaret 112

total war 1, 3-4, 6-8, 10, 12, 17-19, 25-26, 35-37, 43, 58, 63-64, 77-78, 85, 106, 109, 125, 129, 143, 153-154, 158, 160, 169, 187-190, 198

totalitarianism $8,10,19,33,39,81,116$, 147-148, 155

Treaties of Rome 71, 78, 84-85, 175

Treaty of Amsterdam 91 


\section{Index}

Treaty of Maastricht see Treaty on European Union

Treaty of Versailles 35, 65

Treaty on European Union (TEU) 91, 118,149

Turkey $122-124$

UK (United Kingdom) 2, 14, 15, 17, 54, 79, 85-88, 99, 109-110, 112 , 125-129, 187-188, 190

Ukraine 123-124, 130, 190

US (United States) 18, 52, 57-59, 62, 82-83, 85-87, 98, 107, 109, 117, 154-156, 169-183, 188, 196

USSR (United Soviet Socialist Republics) 12, 75, 94-96, 98, 110-111, 114, 116, 124,152
Varoufakis, Yannis 193

Verheugen, Günther 111

Vichy Regime,Vichy France 25, 29, 86, 77, 81, 93

Victory Program 57-58

Waldheim, Kurt 95

Wałęsa, Lech 112

Weimar Republic 35, 145

Wilders, Geert 127-128

Wilson, Woodrow 54

Yanukovych, Viktor 123

Yugoslavia 115, 121-122, 124

Žižek, Slavoj 115 